\title{
Development of Crystal Violet encapsulation in pectin - Arabic gum gel microspheres
}

\author{
Mariana V. Revuelta ${ }^{\text {a }}$, M. Elizabeth Chacon Villalba ${ }^{\text {b }}$, Alba S. Navarro ${ }^{c, d}$, \\ Jorge A. Güida ${ }^{\mathrm{b}, \mathrm{c}, \mathrm{e}}$, Guillermo R. Castro ${ }^{\mathrm{a}, *}$ \\ a Laboratorio de Nanobiomateriales, CINDEFI, Facultad de Ciencias Exactas, Universidad Nacional de La Plata - CONICET (CCT La Plata), 1900 La Plata, Argentina \\ b CEQUINOR, Facultad de Ciencias Exactas, Universidad Nacional de La Plata - CICPBA - CONICET (CCT La Plata), CC 962 , 1900 La Plata, Argentina \\ c Facultad de Ingeniería, Universidad Nacional de La Plata, 1900 La Plata, Argentina \\ d CIDCA, Facultad de Ciencias Exactas, Universidad Nacional de La Plata - CONICET (CCT La Plata), 1900 La Plata, Argentina \\ e Departamento de Ciencias Básicas, Universidad Nacional de Luján, Luján, Argentina
}

\section{A R T I C L E I N F O}

\section{Article history:}

Received 11 April 2016

Received in revised form 18 June 2016

Accepted 10 July 2016

Available online 11 July 2016

\section{Keywords:}

Crystal Violet

Pectin

Arabic gum

Microencapsulation

Ionotropic gelation

Vibrational spectroscopies

Rheological studies

\begin{abstract}
A B S T R A C T
Six pectins with $35 \%$ to $91 \%$ methoxylation degree were purified and characterized to encapsulate Crystal Violet (CV). Amidated low methoxylated pectin (ALMP) was selected based on microsphere morphologies, aqueous solubility, viscosity and the effect of calcium concentration. Pectin microspheres were stabilized with Arabic gum (AG) and optimized according to the loading. Microspheres composed of 2.0\% ALMP-1.0\% AG crosslinked with $450 \mathrm{mM}$ calcium(II) were able to encapsulate $217 \pm 2 \mu \mathrm{M}$ CV. Optical microscopy of the gels revealed spheroid microspheres with $250 \pm 50 \mu \mathrm{m}$ diameter containing homogenous CV distribution. Dried microspheres observed by SEM and epifluorescence showed a highly shrinkable matrix keeping the spheroidal morphology. Low relative viscosity of the ALMP-AG-CV solutions was found compared to ALMP and ALMP-AG. The Young moduli (60-80 Pa) of ALMP-AG microspheres by texturometric analysis indicated that the CV could interfere with the gel crosslinking. Raman spectroscopy analysis suggested some interaction of CV nucleophilic center within the matrix. FTIR of the matrix showed largest shifts in the carbonyl and carboxylate groups probably associated to H-bridges. CV stability studies performed on ALMP-AG microspheres, synthetized from polymer solutions with $\mathrm{pH}$ values above and below pectin $\mathrm{pKa}$ and showed faster $\mathrm{CV}$ release rates in presence of ionic strength.
\end{abstract}

(c) 2016 Elsevier B.V. All rights reserved.

\section{Introduction}

Microencapsulation is becoming popular in the food and pharmaceutical industries, because of preserving physicochemical and biological properties of the load from harsh environments, increasing the solubility of hydrophobic molecules, extending the half-life of the product and also providing molecular controlled release in a proper place and time [1,2]. Some biopolymers can be used to make smart matrices sensitive to diverse environmental conditions (e.g., $\mathrm{pH}$, ionic strength, etc.). Polysaccharides such as alginates, pectins, and natural gums have excellent properties such as biocompatibility, biodegradability, muco-adhesiveness, and sensitivity to many environmental factors. Particularly, pectins and Arabic gums (AG) are considered GRAS (generally regarded as safe, FDA) and used in many industrial applications from foods to pharmaceuticals [2].

\footnotetext{
* Corresponding author.

E-mail address: grcastro@gmail.com (G.R. Castro).
}

Pectins (Pecs) are plant biopolymers soluble in aqueous media and not hydrolysable by human intestinal enzymes but degradable by the microbial community present in the intestine. Pec backbone is composed of poly[ $\alpha$-(1,4)-D-galacturonic acid] partially methoxylated with a pKa in the range of 3.5-4.0 and could contains branches of neutral saccharides. The Pecs can be classified based on the methoxylation degree in: low (0-40\%), medium (40 to 60\%) and high (higher than $60 \%$ methoxylation. Low and medium methoxylated Pecs can make reversible gels in the presence of multivalent cations, while high methoxylated Pecs are forming irreversible gels in presence of solutes (e.g., sugars) under acidic $\mathrm{pH}$ [2]. Amidated low methoxylated pectin (ALMP) are advantageous compared to low methoxylated pectins since can make gels in presence of low bivalent ion concentrations, are less sensitive to $\mathrm{pH}$, and enhanced mucoadhesiveness because of the presence of amide groups [3].

Arabic gum (AG) is a complex biopolymer composed at least of three high-molecular weight fractions extracted from exudates of Acacia trees used in foods. The main fraction of AG is an arabinogalactan-protein complex backbone composed of poly[ $\beta$ - $(1,3)$-D-galactopyranose] with side chains of $\beta$-(1,6)-D-galactopyranose ending with $\beta$-D-glucuronic 
acid and 4-0-methyl- $\beta$-D-glucuronic acid, also branched with $\alpha$-(1,3)-Lrhamnofuranose and $\alpha$ - $(1,4)$-L-rhamnopyranose. The presence of glucuronic acids in AG is responsible of the polyanionic character with a pKa of about 2.0 [4]. The protein containts 18 amino acids with $>50 \%$ composed of hydroxyproline, proline and serine [5]. The simultaneous presences of polar and hydrophobic amino acids are providing the amphiphilic character of the AG able to interact with both hydrophilic and hydrophobic chemical residues of different molecules [2]. The AG relative viscosity is $\mathrm{pH}$-dependent and increases $>100 \%$ from $\mathrm{pH} 2$ to 6 . Also, AG shows Newtonian behavior in aqueous solutions below $40 \%$ (W/V) but pseudoplastic comportment at higher concentrations (shear thinning). In fact, AG is commonly used as emulsifierstabilizing agent of colloidal solutions, and it has low oxygen permeability. Additionally, AG is soluble in aqueous media but gelled at high concentrations [2].

Besides of pectin high solubility in aqueous media, the structural characteristics of the pectin gel matrices crosslinked with multivalent cations sometimes are compromised at $\mathrm{pH}$ above the pectin $\mathrm{pKa}$, present in physiological environments (i.e., intestine). The simplest and greenest solution is to make coacervates, i.e., undefined chemical mixture of polymers. Arabic gum was selected for the coacervate formulation because of its gelling properties, biocompatibility, absence of toxicity in their degradation products and no-immunogenic response. However, the main requisite of coacervate synthesis is the chemical compatibility between the polymer components mainly established by short-term bond interactions (i.e., Hydrogen bonding, Van der Waals forces and others) which are stabilizing the matrix structure against different environments [6]. Examples of pectin coacervates showing proper molecular controlled release were previously reported [7].

Crystal Violet (CV) is a synthetic dye well known in the identification of bacteria by the Gram staining technique but also used in foods, cosmetic and fabrics (Fig. 1). More importantly, CV was pharmacologically employed for the treatment of fungi, parasites (i.e., leishmania, trypanosomes, etc.) and microbial infections in humans since the XIX century. However, many undesirable side effects were reported by therapeutic use of CV in biological systems such as mutagenic, carcinogenic, necrotic skin effects, irritant of mucosal membranes and depression of white blood cell lines because of the high doses of the dye [8]. One of the main problems associated with the administration of $\mathrm{CV}$ is related to the molecular aggregation in aqueous media that could probably be attributed to the $\pi-\pi$ stacking of the aromatic rings. The CV aggregation is related to the concentration, at very low dye concentrations, below $1 \mathrm{mM}$, the dimerization is the main mechanism defined by a thermodynamic equilibrium constant of $K_{\mathrm{d}}=6 \times 10^{-2} 1 \cdot \mathrm{mol}^{-1}$ at $20^{\circ} \mathrm{C}$, but at high CV concentrations multimeric aggregates were reported [9]. In previous work, CV aggregation in aqueous media was prevented by the addition of the poly(methacrylic acid) to the solution [10]. The complex formation was attributed to high hydrophobicity of poly(methacrylic acid) which provided a rigid matrix for CV exclusion from the aqueous polar medium and enhanced dye reactivity.

$\mathrm{CV}$ encapsulation and entrapment in matrices such as particles made of acrylamide and acrylates derivatives or in magnetic zeolite nanoparticles and in sol-gel silica clusters were reported [9,11,12]. However, the mentioned systems are requiring chemical synthetic precursors (i.e., acrylamide, tetraethoxysilane) or materials produced by coal combustion (i.e., fly ash) which are not environmentally friendly. Also, CV was encapsulated in liposomes made of phospholipids and dicetyl phosphate and SDS micelles, in both methods organic solvents are used [13, 14]. Those lipid structures are thermodynamically unstable.

On the other hand, novel trends in matrix developments using green technologies are gaining more adepts in the academy and industry. For example, polysaccharides are no toxic, no immunogenic, biodegradable and biocompatible, can make 3-D structures by soft techniques like ionotropic gelation techniques in presence of divalent ions or by selfassembly make them very suitable hydrogels for medical applications such as drug delivery [15]. Hydrogel microencapsulation is an alternative to avoids adverse effects posed by the load and release of small molecules such as instability, bioavailability and toxicity. Biopolymeric microparticles are of special interest for oral drug delivery because of their stability, small size, porous control, tailoring, large surface area and safety, favoring their absorption and controlling the load release kinetic compared to other molecular carriers [16].

The aim of the work was to study and characterize the encapsulation of Crystal Violet in a biopolymeric matrix composed of pectin and Arabic gum. Pectins with different methoxylation degrees were purified followed by interaction studies between the dye and the biopolymers. Amidated low methoxylated pectins were chemically characterized and used to produce gel microspheres containing the dye. The microspheres were characterized by optical, epifluorescence and scanning electron microscopies (SEM). Mechanical, rheological and vibrational spectroscopies (FITR and Raman) were used to establish the hybrid matrix properties and the interactions between their components and the Crystal Violet. CV stability studies of microspheres synthetized from polymer solutions with $\mathrm{pH}$ values above and below pectin $\mathrm{pKa}$, in presence or absence of ionic strength, were performed to examine the effect on CV encapsulation and its kinetics release.

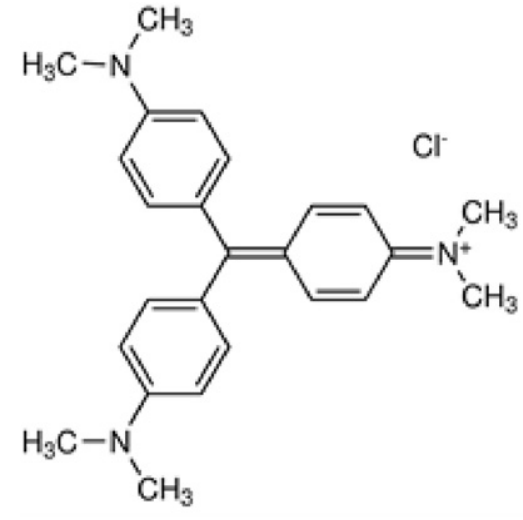

A

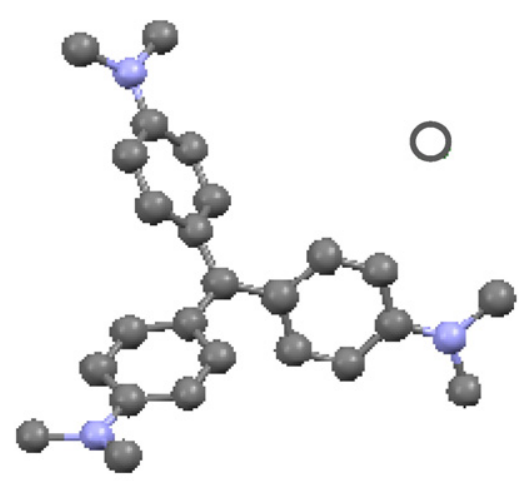

B

Fig. 1. Geometrical arrangement of Crystal Violet (A) and molecular structure showing the propeller geometry of the dye (B). 


\section{Materials and methods}

\subsection{Materials}

Pectins from citric and apple with low and high methoxylation degree were under study and purchased from Sigma-Aldrich (Buenos Aires, Argentina) or kindly provided by Gelfix or CPKelco (Buenos Aires, Argentina). Amidated low methoxylated pectin (ALMP) was kindly provided from Gelfix, Arabic Gum (AG), Crystal Violet (CV, 4\{bis[4-(dimethylamino)phenyl]methylidene\}-N,N-dimethylcyclohexa2,5-dien-1-iminium, MW = 407.98) and buffers were purchased from Sigma-Aldrich (Buenos Aires, Argentina). Other reagents and solvents were of analytical grade from Merck (Darmstadt, Germany).

\subsection{Crystal Violet spectrophotometric analysis}

CV stock solution (1.0 mM) and its dilutions were made in $10 \mathrm{mM}$ TES buffer ( $\mathrm{pH}=7.4$.) and the calibration curve was performed at $\lambda_{\max } 592 \mathrm{~nm}\left(\bar{v}=16,900 \mathrm{~cm}^{-1}\right)$ in a UV-vis spectrophotometer (Beckman DU 640, Beckman-Coulter, CA, USA). The CV extinction coefficient of $\varepsilon=9.8 \times 10^{4} \mathrm{M}^{-1} \mathrm{~cm}^{-1}$ at the maximum absorption was used for the calculations as previously reported [17].

\subsection{Pectin purification and characterization}

Pectins were purified by precipitation from aqueous solution using cold ethanol at $4{ }^{\circ} \mathrm{C}$. Cold ethanol was added to a solution containing $20-40 \mathrm{~g} \cdot \mathrm{l}^{-1}$ of pectin to reach $70 \%-80 \%(\mathrm{v} / \mathrm{v})$. The suspension was centrifuged at $6,000 \times \mathrm{g}$ for $20 \mathrm{~min}$ at $4{ }^{\circ} \mathrm{C}$ and the supernatants were analyzed for sucrose content by HPLC. Also, Pec samples were analyzed for sucrose content by HPLC run isocratically with water at $0.2 \mathrm{ml} \cdot \mathrm{min}^{-1}$ in C8 column (Shodex, USA) and detected by IR (at internal/external temperatures $45^{\circ} \mathrm{C} / 80^{\circ} \mathrm{C}$ ) using appropriate calibration curve. The procedures were repeated until no sucrose was detected. Later the pectin samples were dissolved in water and dialyzed (5000 MWCO membranes, Millipore, Billerica, Ma) against MilliQ water and freeze dried.

Pectin methoxylation degree was determined by acid-base titration method: $50 \mathrm{mg}$ of Pec was dissolved in $10 \mathrm{ml}$ of water containing phenolphthalein as acid-base indicator, and titrated with $500 \mathrm{mM} \mathrm{NaOH}$ $\left(\mathrm{V}_{1}\right)$. Later, $1.00 \mathrm{ml}$ of $500 \mathrm{mM} \mathrm{NaOH}$ was added to the solution and kept for $15 \mathrm{~min}$ at room temperature, followed by the addition of $1.00 \mathrm{ml}$ of $500 \mathrm{mM} \mathrm{HCl}$. Finally, the $\mathrm{HCl}$ acid excess was titrated with $500 \mathrm{mM} \mathrm{NaOH}\left(\mathrm{V}_{2}\right)$. The methoxylation percentage was estimated by the following equation:

Methoxylation $(\%)=\frac{V_{2}}{V_{1}+V_{2}} \times 100$

The galacturonic acid content in pectin was determined spectrophotometrically at $650 \mathrm{~nm}$ using 3,5-dimethyl phenol [18].

\subsection{Viscosity analysis}

Aqueous solutions of $0.02 \%$ to $0.12 \%$ (p/v) CV, ALMP-AG, ALMP-AG$\mathrm{CV}$ were determined by Ostwald viscometer (Serie 100, USA) and rotational viscometer (Haake VT 550, Germany). The results were analyzed by the Power Law [19]:

$\sigma_{\theta r}=-m\left(d v_{\theta} / d r\right) \mathrm{n}$

where $\sigma_{\theta r}$ is shear stress $(\mathrm{Pa}), d v_{\theta} / d r$ is shear rate $\left(\mathrm{s}^{-1}\right), m$ is consistency index $\left(\mathrm{Pa} * \mathrm{~S}^{\mathrm{n}}\right), \mathrm{n}$ is a dimensionless flow behavior index $(0<\mathrm{n}<1$ for pseudoplastic fluids).

\subsection{Biopolymer formulation and microsphere formation}

$\operatorname{ALMP}(2.0 \%, 1.5 \%$, and $1.0 \%, \mathrm{w} / \mathrm{v})$ and $\operatorname{AG}(1.00 \%, 0.75 \%$, and $0.50 \%, \mathrm{w} /$ v) solutions were made in milliQ water (Millipore, Billerica, MA, USA). Formulations of ALMP-AG solutions containing the dye were prepared by the slow dropping of $245 \mu \mathrm{M} \mathrm{CV}$ and stirred at room temperature for $12 \mathrm{~h}$.

Hydrogel microspheres were made by extrusion of the biopolymer solution through a $100 \mu \mathrm{m}$ diameter syringe coupled with a peristaltic pump (Watson-Marlow, UK). Biopolymer coacervates with and without $\mathrm{CV}$ were dropped into a solution containing 450 or $900 \mathrm{mM} \mathrm{CaCl}_{2}$ at $0{ }^{\circ} \mathrm{C}$ under continuous stirring to avoid coalescence of gel microspheres. Also, ALMP-AG (2.0\%-1.0\%) gel microspheres were synthesized from polymer solutions at $\mathrm{pH} 3.0$ (20 mM citrate buffer) and 5.0 (100 mM acetate buffer) dropped into $450 \mathrm{mM} \mathrm{CaCl}_{2}$. Microspheres were aged in the $\mathrm{CaCl}_{2}$ solution for $20 \mathrm{~min}$, followed by filtration on paper (Whatman \#1). Filtered microspheres were kept in $450 \mathrm{mM} \mathrm{CaCl}_{2}$ solution containing $10 \mu \mathrm{M} \mathrm{NaN}{ }_{3}$ at $5{ }^{\circ} \mathrm{C}$. Alternatively, microspheres were air-dried at room temperature and lyophilized.

\subsection{Optical and scanning electron microscopies}

Optical microscopy of the microparticles loaded with CV were performed in epifluorescence microscope (Leica DM 2500, Germany) equipped with UV filters (495-505 nm).

Scanning Electron Microscopy (SEM) analysis was carried out in freeze-dried microparticles. Microsphere samples were prepared by sputtering the surface with gold (Balzers SCD 030 metalizer) obtaining layer thickness between 15 and $20 \mathrm{~nm}$. Microparticles surfaces and morphologies were observed using Philips SEM 505 model (Rochester, USA), and processed by an image digitalizer program (Soft Imaging System ADDA II (SIS)).

\subsection{Measurement of gel mechanical parameters}

Microspheres texture was analyzed in a TAXT2i Texture Analyzer (Stable Micro Systems Ltd., UK) equipped with a load cell of $25 \mathrm{~kg}$ and a cylindrical metal compression plate of $75 \mathrm{~mm}$ diameter (p75). The Texture Profile Analysis (TPA) consisted in two compression cycles at $0.5 \mathrm{~mm} \mathrm{~s}^{-1}$ to $50 \%$ of the original microspheres height with a $2 \mathrm{~s}$ gap between cycles. The parameters obtained by the texturometer were corrected following previous report [20]. "True strain" is showing the extension of the deformation and expressed by $\ln \left(\mathrm{L}_{\mathrm{o}} / \mathrm{L}\right)$, where $\mathrm{L}_{\mathrm{o}}$ and $\mathrm{L}$ are the high before and after compression respectively. "True stress" is the stress corrected for the enlargement of the mean cross-sectional area of the sample during the compression and it is given by $[\mathrm{F} /$ $\left.\mathrm{A} \times\left(\mathrm{L} / \mathrm{L}_{0}\right)\right]$, where $\mathrm{F}$ is the rupture force and $\mathrm{A}$ the initial cross-section area of the sample. Young's modulus, related to the network firmness, was calculated from the slope of the true stress vs. true strain curve.

\subsection{Infrared and Raman instrumentation}

The infrared spectra of the substances as $\mathrm{KBr}$ pellets were recorded in the 4000 to $400 \mathrm{~cm}^{-1}$ range and scanned with background correction at 60 scans on a Bruker Equinox 55 FTIR spectrometer. The Raman spectra of the solids, run in the region between 3500 and $100 \mathrm{~cm}^{-1}$, were obtained with a FRA 106 accessory mounted on a Bruker IFS 66 FTIR instrument, using the $1064 \mathrm{~nm}$ excitation line from an Nd-YAG laser. Infrared and Raman spectra were scanned with $4 \mathrm{~cm}^{-1}$ resolution.

\subsection{Stability studies of microencapsulated Crystal Violet}

ALMP-AG (2.0\%-1.0\%) gel microspheres ( $50 \mathrm{mg}$ ) were suspended in $2.0 \mathrm{ml}$ aqueous buffers on rotary shaker at $100 \mathrm{rpm}$ and $37^{\circ} \mathrm{C}$. In vitro $\mathrm{CV}$ kinetic release were studied at $\mathrm{pH} 1.2(20 \mathrm{mM} \mathrm{KCl} / \mathrm{HCl})$, and at 7.4 (100 mM buffer TES). All assays were performed in the presence or 
absence of $154 \mathrm{mM} \mathrm{NaCl}$. Samples of $1.0 \mathrm{ml}$ were taken out from the vials and the CV released from the microspheres was spectrophotometrically determined at defined time intervals. The volume of the vials was kept constant by replenish with $1.0 \mathrm{ml}$ of fresh media.

\section{Results and discussion}

\subsection{Pectin purification and characterization}

Commercial pectins, usually used in foods could contain $>20 \%$ sucrose. In consequence, the development of biopolymer purification is required for the purposes material and matrix characterizations, gel crosslinking, and controlled release studies. The common and simplest procedure for polymer purification is precipitation which commonly involves the use of kosmotropic salts (e.g., ammonium sulfate) and/or organic solvents (e.g., acetone or ethanol). Besides, organic solvents are preferred because of no further purification steps are required.

Six purified pectins containing from $35 \%$ to $91 \%$ methoxylation degree were screened for microspheres gel formation and CV encapsulation in presence of $200 \mathrm{mM} \mathrm{Ca}^{2+}$ or $\mathrm{pH} 2.0$ containing of solute (i.e., glucose) for low and high methoxylated polymers respectively. Similarly, pectin concentrations from $1.0 \%$ to $4.0 \%$ were studied to obtain microspheres containing CV. However, high pectin concentrations are making very viscous colloidal solutions difficult to manipulate, also in some cases microsphere aggregation and irregular gel morphologies during formation was observed especially using high methoxylated pectins (data not shown). In consequence, low methoxylated pectin and amidated low methoxylated pectin (LMP and ALMP respectively) were selected for further studies also because of high aqueous solubility and low relative viscosity compared to HMP (data not shown). Analysis of both low methoxylated pectins showed similar degree of methoxylation (i.e., 35-40\%) but the amount of galacturonic acid is about 24\% higher in LMP compared to the ALMP because of the $20 \%$ amidation degree is reducing the amount of free carboxylate residues (Table 1S, Supplemental material). Later, the effect of calcium concentration on the turbidity of polymer solutions was taken into account for the selection of LMP (Fig. 2). The increase of calcium ions into the polymer solutions reduced the turbidity for both LMPs at minimum values in the range of $500 \mathrm{mM}$ to $900 \mathrm{mM}$, after that region the turbidity increased again in both pectin solutions. These results are related to the interaction between the $\mathrm{Ca}^{2+}$ and the free carboxylate groups of the pectins which could suggest polymer sol-gel transitions as previously reported [7]. The decrease of the turbidity at low calcium concentration

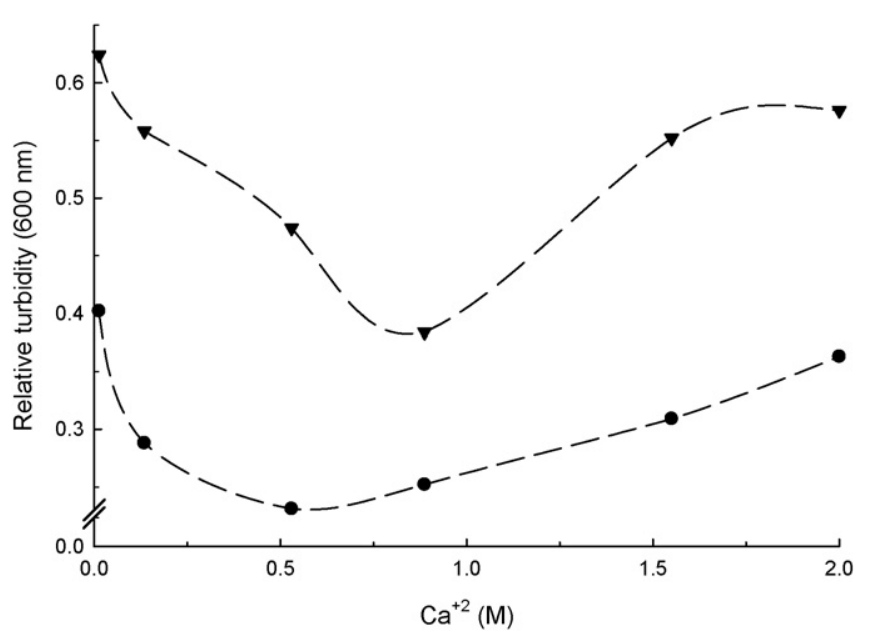

Fig. 2. Effect of calcium concentration on polymer aggregation of low methoxylated ( $)$ and amidated low methoxylated $(\boldsymbol{\nabla})$ pectins. until a minimum value is reached can be attributed to the interaction of $\mathrm{Ca}^{2+}$ with the Pec chains making the sol-gel transition by increasing the interaction between pectin chains mediated by calcium. The increase of $\mathrm{Ca}^{2+}$ concentrations higher than $500-900 \mathrm{mM}$ is enhancing the turbidity that could be attributed to the electrostatic repulsion among the calcium-protonated pectin chains. Particularly, LMP solution possess less initial turbidity than ALMP but the relative changes of turbidity produced by the increase of calcium concentration in LMP are lower compared to the ALMP. The behavior of LMP related to the changes of $\mathrm{Ca}^{+2}$ concentrations could be addressed to the high amount of free galacturonic acid in the polymer chains compared to the ALMP. Considering the relative sensitivity of ALMP to the environmental conditions mediated by calcium concentrations, it was selected for further experiments. The high relative sensitivity of ALMP to calcium ion concentrations could be linked to the presence of lone electron pair of Nitrogen from the amide groups that could be able to interact with $\mathrm{Ca}^{2+}$, but without the strength of the carboxylate interactions. The presence of amide groups in ALMP makes the biopolymer more hydrophobic and consequently the trend to form gels increases producing more resistant $3 \mathrm{D}$ architectures in aqueous media $[3,21]$.

\subsection{Microsphere formation and properties}

Gel microspheres made of 2.0\% ALMP by ionotropic gelation with 113 to $900 \mathrm{mM}$ calcium range encapsulated from $88 \%$ to $94 \%$ CV (data not shown). However, microspheres showed high swelling when the pectin gels were placed in contact with solutions at $\mathrm{pH}$ above the $\mathrm{pKa}$ of the biopolymer [7]. Therefore, alternatives to prevent high gel swelling keeping the matrix structure and controlling the molecular release of the load are required. One of the simplest and greenest strategies is to make coacervates using polymers having different properties. Arabic gum was selected because of its properties of good solubility combined with low viscosity in aqueous media and no-toxicity [22].

ALMP-AG coacervates made of different polymer ratios were tested for gelling capacity in presence of $450 \mathrm{mMCa}^{2+}$ and the best ratio to obtain spheroidal and homogeneous gel structures was found at 2:1 ratio (data not shown). The next step was to adjust the concentration of the ALMP:AG (2:1) for CV encapsulation. The CV concentration was established on $245 \mu \mathrm{M}$ to avoid molecular stacking in aqueous media [17]. ALMP-AG formulations composed of $2.0 \%-1.0 \%, 1.5 \%-0.75 \%$ and $1.0 \%-0.5 \%$ were able to encapsulate $88.5 \%, 77.3 \%$ and $67.0 \%$ of $C V$ respectively in presence of $450 \mathrm{mM} \mathrm{Ca}^{2+}$.

Morphological analysis of gel microspheres by optical microscopy showed spheroidal structure and sharp borders with average diameter of about $200 \pm 30 \mu \mathrm{m}$ in all cases (Fig. 3). However, microsphere morphologies and size distribution was compromised at $1.50 \%-0.75 \%$ or lower ALMP-AG concentrations under our experimental conditions. Besides, because of the $88.5 \% \mathrm{CV}$ load in the microspheres, the $2.0 \% \mathrm{ALMP}$ $1.0 \%$ AG formulation was selected for further studies considering spherical morphology and size homogeneity (Table 2S, Supplementary material).

ALMP-AG (2.0\%-1.0\%) gel microspheres were synthesized at pH 3.0 and $\mathrm{pH} 5.0$, values below and above of pectin pKa, to evaluate the effect on CV encapsulation. CV encapsulation on the gel microspheres performed at $\mathrm{pH} 3.0$ and $\mathrm{pH} 5.0$ showed of $74.0 \%$ and $76.0 \%$ of dye entrapment respectively (Table 3S, Supplementary material). These results are indicating no significant effects of the polymer solution $\mathrm{pH}$ on the dye encapsulation process.

SEM images of 2.0\% ALMP - 1.0\% AG dried microspheres showed a highly shrinkable structure but without losing the structural main morphology (Fig. 4). Comparative analysis of optical microscopy and SEM images of microspheres are suggesting the high water content in the ALMP-AG biogel. Also, the microscopy images showed smooth and homogenous surface of microspheres without substantial deep cracks indicating a uniform microstructure without polymer segregation. This 

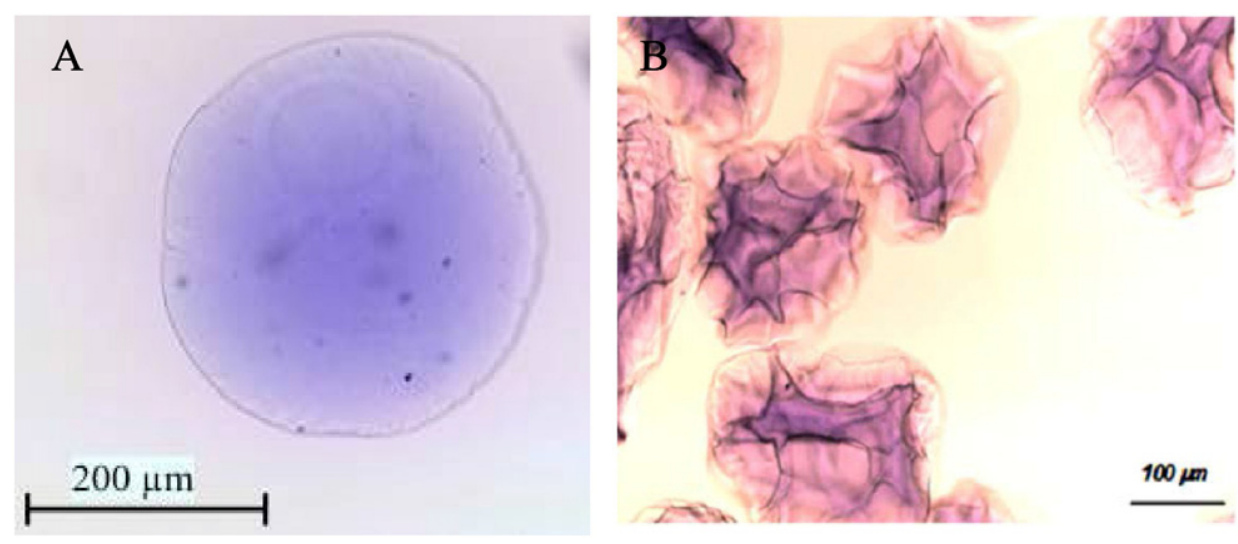

Fig. 3. Optical microscopy $(100 \times)$ of ALMP-AG gel microspheres containing CV. Native $(A)$ and freeze-dried (B) microspheres respectively.

result is relevant because of suggesting a good interpenetration network made between ALMP and AG under our experimental conditions.

\subsection{Viscosity analysis}

Rheological analysis of ALMP, AG, and ALMP-AG coacervates with and without CV are shown in Table 1. ALMP and AG are displaying pseudoplastic $(\mathrm{n}<1)$ and Newtonian $(\mathrm{n} \approx 1)$ behaviors respectively. However, ALMP-AG coacervates are showing intermediate behavior, but more close to Newtonian fluids when CV was added to the colloidal solution. This result was evidenced by the consistency index about 8 times lower compared to the ALMP-AG formulation without the dye.

The curves of apparent viscosity versus shear rate shown in Fig. $5 \mathrm{~A}$ were very close for ALMP and AG similarly as previously reported [22]. The apparent and relative viscosities of ALMP solutions are higher than the AG probably because of the polar content provided by the amide and carboxylate free groups able to interact with the aqueous solvent and trapped in high quantities inside the gel which is increasing the viscosity of the solution [23]. The low viscosity of AG can be attributed to the high branched molecular structure of the biopolymer with high interaction between the side chains which induce to a more compact molecular arrangement of polymer reducing the water solvation [24]. Besides, the relative viscosity of ALMP and its coacervates rose with the total polymer concentration (Fig. 5B), with the exception of AG which displayed Newtonian behavior along the tested concentrations.

Addition of CV to the biopolymer coacervate reduces the apparent viscosity of the system suggesting some molecular interactions between the dye and the biopolymers.
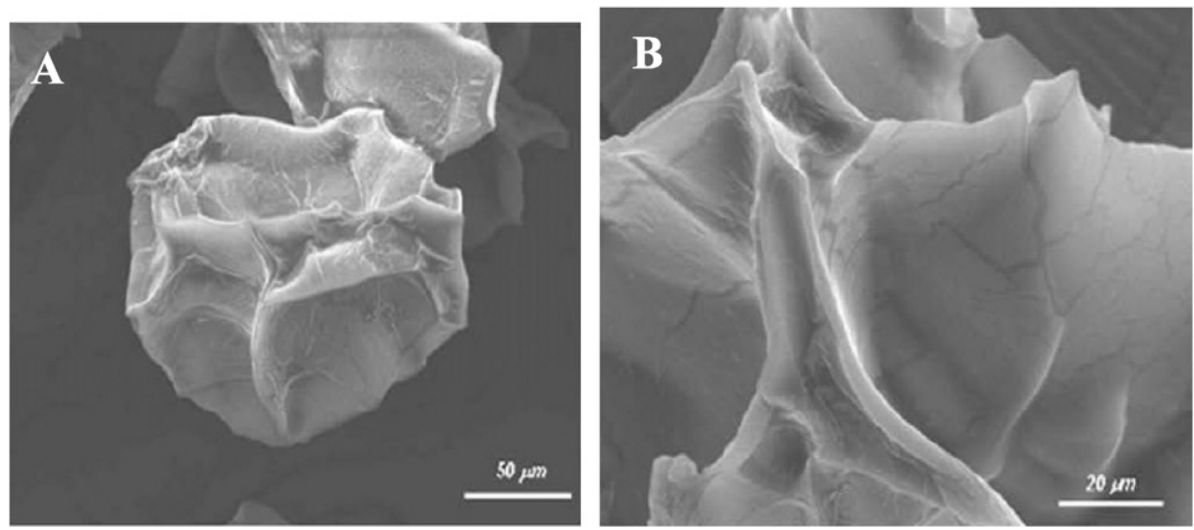

Fig. 4. Scanning electron microscopy of ALMP-AG gel microspheres containing Crystal Violet. 
Table 1

Consistency index (m) and flux index (n) of the formulations.

\begin{tabular}{lll}
\hline Formulation & $m\left(\right.$ Pa. $\left.^{n}\right)$ & $n$ \\
\hline ALMP & $0.180 \pm 0.004$ & $0.746 \pm 0.008$ \\
AG & $0.006 \pm 0.002$ & $1.060 \pm 0.065$ \\
ALMP-AG & $0.103 \pm 0.004$ & $0.831 \pm 0.031$ \\
ALMP-AG-CV & $0.023 \pm 0.001$ & $1.045 \pm 0.011$
\end{tabular}

Obs.:Values are the mean \pm SD

providing complementary information of the microspheres components. CV bands are not observed in infrared spectra because of the dye low concentration. Conversely, the strong dispersion observed for $\mathrm{CV}$ in Raman provided good quality spectra even in the low concentration level of CV in the matrix. Then, infrared spectroscopy revealed interactions between matrix components while Raman evidenced interactions between CV and the formulation.

The Raman spectra of $220-230 \mu \mathrm{M}$ CV and the formulation are compared in Fig. 6. In order to analyze bands details only selected spectral regions are shown. The CV bands observed in the spectra compares favorably with that reported before by other authors [25], but in present case with a better signal noise ratio, possible due to the infrared laser beam light used at $1064 \mathrm{~nm}$. Besides, the Raman spectrum of formulation is very similar to the CV spectra. This fact evidences the strong dispersion shown by the dye in contrast with the relatively poor response observed for polysaccharides in the formulation, even in larger concentration. Wavenumbers and assignments for Raman spectra of CV and formulation are collected in Table 2.

Nevertheless, the band at $853 \mathrm{~cm}^{-1}$ observed in the formulation is attributed to $v$ (C6-C5-05-C1-01) mode of ALMP. The band at $2940 \mathrm{~cm}^{-1}$ is assigned to the aliphatic $\nu(\mathrm{CH})$ vibrations. The relative intensity of this band in the formulation is enhanced respect to the $\mathrm{CV}$ spectrum, probably due to the contribution from the polysaccharides. It should be noted that $v(\mathrm{CH})$ is one of the most intense bands in the Raman spectrum of polysaccharides (Fig. 2S, Supplementary material).

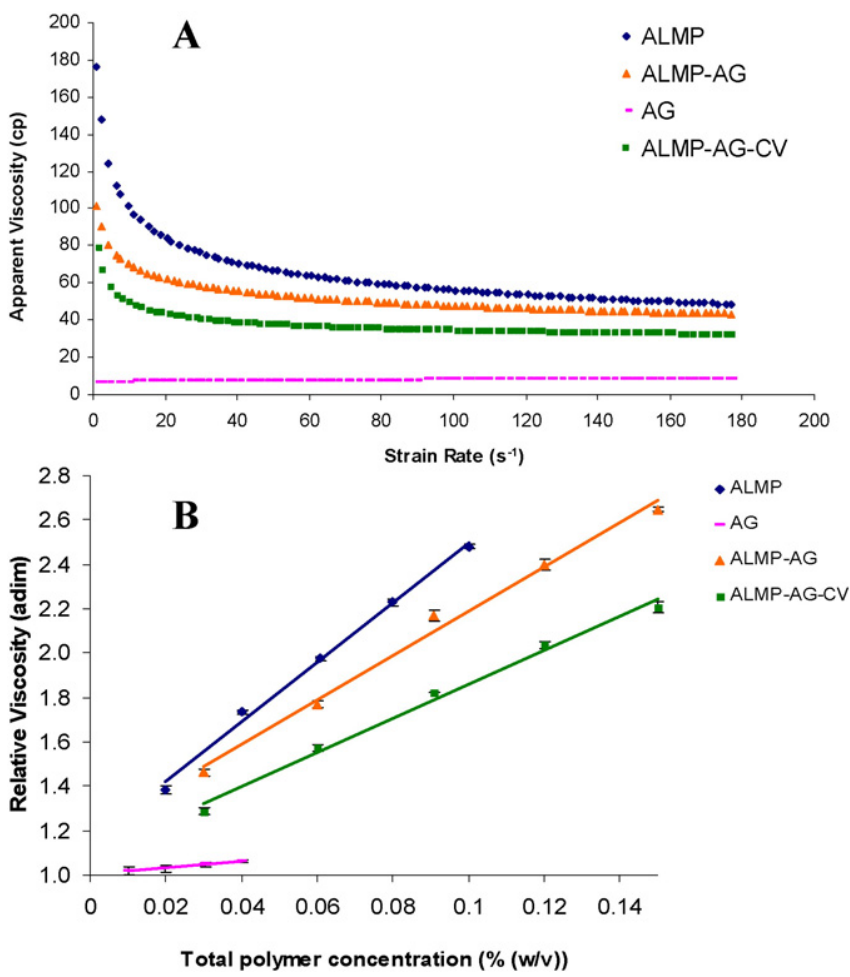

Fig. 5. Apparent viscosity vs. strain rate (A) and relative viscosity vs. total polymer concentration (B) for ALMP, AG, ALMP-AG and ALMP-AG-CV at $25^{\circ} \mathrm{C}$. All experiments were performed in duplicates and the graph is showing the mean $\pm \mathrm{SD}$.

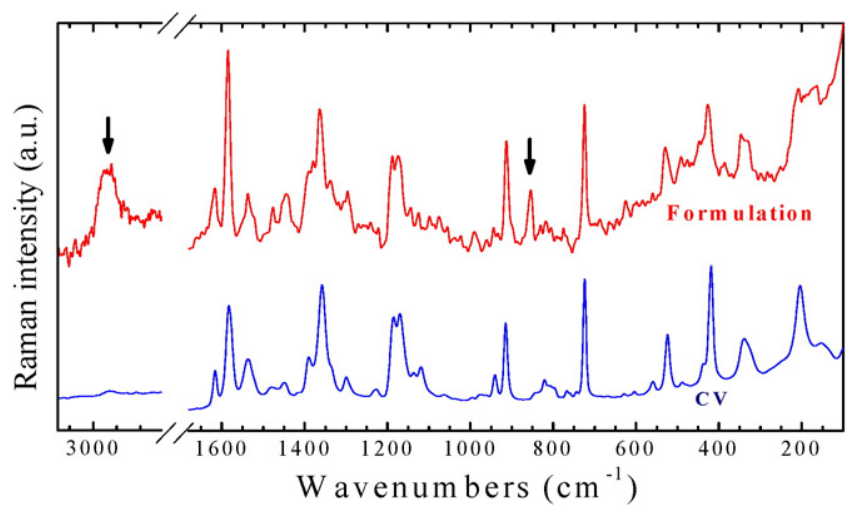

Fig. 6. Raman spectra of CV and the formulation composed of CV in ALMP-AG. Bands indicated with arrows came from the polysaccharides (see text).

In Table 2, the assignments of pure CV and CV in the matrix are displayed with an additional column containing spectroscopic shifts (defined by $\Delta=$ wavenumber of $C V$ in the matrix $(F)-C V$ wavenumber). It can be seen that not all the CV bands in the matrix were shifted respect the $\mathrm{CV}$ frequencies. According to the $\Delta$ definition, positive signs indicate bonds reinforcement when the dye is in the formulation, while negative shift means weaker ones. The maximum shift registered in this work was $8 \mathrm{~cm}^{-1}$. This value may be considered relatively small in comparison with that observed by other dye in a similar formulation [26]. In the present work, it is assumed that the greater shifts of a given mode the greater is the interaction between dye and formulation. Therefore, Raman spectroscopy applied to this system is adequate to evaluate interactions between the dye and the formulation, but is unable to evaluate polymers components interactions because their bands are too weak to be observed in Raman in the same spectrum.

Table 2

Raman assignments for Crystal Violet and formulation bands.

\begin{tabular}{|c|c|c|c|}
\hline CV & $\mathrm{F}$ & $\Delta(\mathrm{F}-\mathrm{CV})$ & Assignments \\
\hline $2930 \mathrm{w}$ & - & - & $v(\mathrm{CH})_{\mathrm{CH} 3}$ \\
\hline- & $2940 \mathrm{~m}$ & - & $v(\mathrm{CH})_{\mathrm{CH} 3}$ \\
\hline $2816 \mathrm{vw}$ & & & $v(\mathrm{CH})_{\mathrm{N}-(\mathrm{CH} 3)}$ \\
\hline $1616 \mathrm{~m}$ & $1617 \mathrm{~m}$ & & \\
\hline $1583 \mathrm{~s}$ & $1585 \mathrm{~s}$ & +2 & $v(\mathrm{C}-\mathrm{C})_{\text {Ring }}$ \\
\hline $1536 \mathrm{~m}$ & $1537 \mathrm{~m}$ & & \\
\hline $1480 \mathrm{w}$ & $1476 \mathrm{w}$ & -4 & $v(\mathrm{C}-\mathrm{C})_{\text {Ring }}+$ Ring deformation \\
\hline $1449 \mathrm{w}$ & $1445 \mathrm{w}$ & -4 & \\
\hline \multirow[t]{2}{*}{$1390 \mathrm{~m}$} & 1381 & - & $v(\mathrm{~N}-\mathrm{Phenyl})$ \\
\hline & 1390 & & \\
\hline 1358 vs & $1364 \mathrm{~s}$ & +6 & \\
\hline $1336 \mathrm{sh}$ & $1340 \mathrm{w}$ & +4 & \\
\hline $1300 \mathrm{~m}$ & $1296 \mathrm{~m}$ & -4 & $v(C-C)_{\text {Ring }}$ \\
\hline $1227 \mathrm{w}$ & - & - & Ring $\mathrm{CH}$ bend ( $\|$ ) \\
\hline 1185 vs & $1188 \mathrm{~s}$ & +3 & \\
\hline $1170 \mathrm{vs}$ & $1175 \mathrm{~s}$ & +4 & \\
\hline 1137 vw & - & - & \\
\hline $1119 \mathrm{w}$ & - & - & \\
\hline $941 \mathrm{~m}$ & - & - & Ring skeletal vib rad orientation \\
\hline $915 \mathrm{~s}$ & $913 \mathrm{~s}$ & -2 & \\
\hline- & 853 & - & $\mathrm{C} 6-\mathrm{C} 5-\mathrm{O} 5-\mathrm{C} 1-\mathrm{O} 1$ (pectin) \\
\hline $821 w$ & - & - & Ring $\mathrm{CH}$ bend $(\perp)$ \\
\hline $760 \mathrm{vw}$ & - & - & \\
\hline 724 vs & 725 vs & +1 & \\
\hline $604 \mathrm{vw}$ & - & - & Ring skeletal vib rad orientation \\
\hline $559 \mathrm{w}$ & $559 \mathrm{vw}$ & 0 & \\
\hline $524 \mathrm{~m}$ & $530 \mathrm{~m}$ & +6 & \\
\hline $437 \mathrm{w}$ & $448 w$ & +11 & Phenyl- $C^{+}$-Phenyl $(\perp)$ bend \\
\hline 419 vs & $427 \mathrm{~s}$ & +8 & \\
\hline $340 \mathrm{~m}$ & $347 \mathrm{~m}$ & +7 & Phenyl-C ${ }^{+}$-Phenyl( $\|$)bend \\
\hline $325 \mathrm{sh}$ & $330 \mathrm{sh}$ & +5 & \\
\hline $205 \mathrm{~s}$ & $209 \mathrm{~s}$ & +4 & Breathing of central bonds \\
\hline
\end{tabular}

Obs.; CV: Crystal Violet; F: Formulation, $\Delta(\mathrm{F}-\mathrm{CV})$ : Spectroscopic shifts $=$ (wavenumber of $\mathrm{CV}$ in matrix ( $\mathrm{F})$ - CV wavenumber). 
Table 2 is showing the shifts larger than $2 \mathrm{~cm}^{-1}$ to avoid instrumental inaccuracies for the well-defined bands and correspond to the following vibrational modes: $v(\mathrm{~N}-\mathrm{Phenyl})(+6) ; v(\mathrm{C}-\mathrm{C})_{\text {Ring }}(-4)$; ring $\mathrm{CH}$ bend $(\mathrm{II})(+4)$; ring skeletal vibration of radical orientation $(+6)$; phenyl- $\mathrm{C}^{+}$-phenyl( $\|$)bend $(+8)$; phenyl- $\mathrm{C}^{+}$-phenyl $(\|)$bend $(+7)$; and breathing of central bonds $(+4)$.

The CV crystal structure, by the form of chloride salt and two hydrates: $\mathrm{CV}^{+} \mathrm{Cl}^{-} . \mathrm{H}_{2} \mathrm{O}$ and $\mathrm{CV}^{+} \mathrm{Cl}^{-} .9 \mathrm{H}_{2} \mathrm{O}$, were reported previously [27]. The CV cation has typical propeller geometry (Fig. 1B). The dihedral angle between the aryl rings and the central coordination plane is about $27^{\circ}$. Therefore the central $\mathrm{C}$ has not chances of interaction with the matrix. The most exposed groups to generate interaction are the amine groups: $-\mathrm{N}\left(\mathrm{CH}_{3}\right)_{2}$.

According to the CV geometry detailed above, the lone electron pair located on the nitrogen atoms of amine groups are the external part of dye exposed for the interaction with polymers. These nucleophilic centers might form hydrogen bond with the $\mathrm{OH}$ groups of polymers. As stronger is the interaction larger will be the frequency required for the corresponding modes. Then, it is expected an increasing frequency of $v(\mathrm{~N}-\mathrm{C}($ phenol $))$ vibration mode when the $\mathrm{CV}$ is included in the formulation. This prediction is in agreement with the observed shift $\left(+6 \mathrm{~cm}^{-1}\right)$ found for CV crystals at $1358 \mathrm{~cm}^{-1}$. This vibrational mode couples strongly with ring vibrations, according to the reference [28], for this reason, the vibrational modes associated with ring is shifted in the formulation. That is the case of ring $\mathrm{C}-\mathrm{H}$ bend( $\|$ ) and ring skeletal vibrational radical orientation modes which are observed in VC at 1185 $(\Delta v=+3)$ and $1170 \mathrm{~cm}^{-1}(\Delta v=+4)$ for the first one and $524 \mathrm{~cm}^{-1}$ $(\Delta v=+6)$ for the second. But the band at $915 \mathrm{~cm}^{-1}$, assigned to a ring skeletal vibrational radical orientation mode, practically keep unshifted (only shift $-2 \mathrm{~cm}^{-1}$ ) (Table 2 ).

The band at $853 \mathrm{~cm}^{-1}$ was assigned to a $\mathrm{C} 6-\mathrm{C} 5-\mathrm{O} 5-\mathrm{C} 1-01$ vibrational mode of pectin. This and that at $2940 \mathrm{~cm}^{-1}$ are the only bands coming from the polymers formulation.

Interestingly, the $\mathrm{CV}$ bands at $419 \mathrm{~cm}^{-1}$ and $340 \mathrm{~cm}^{-1}$, assigned to the deformation $\mathrm{Ph}-\mathrm{C}^{+}-\mathrm{Ph}$, suffer the largest shifts observed in the spectra. These bands shift $+8 \mathrm{~cm}^{-1}$ and $+7 \mathrm{~cm}^{-1}$, respectively in the formulation. Since central atom unlikely form a direct interaction with the matrix, being that this atom is sterically hindered. The positive shift observed could be understood considering that bending modes require higher energies in the formulation than in the free cation because the phenyl groups are anchored to the polymers through ammine groups.

The band at $205 \mathrm{~cm}^{-1}$ in CV spectrum corresponds to the breathing of central bonds. This band is observed at $209 \mathrm{~cm}^{-1}$ in the formulation (Table 2). This shift might be explained in similar way than the former.

In conclusion, the most significant shifts found correspond to the following modes: $v(\mathrm{~N}-\mathrm{C}(\mathrm{pH})$ ), ring skeletal vibrational radical orientation and deformation $\mathrm{Ph}-\mathrm{C}^{+}-\mathrm{Ph}$. Therefore, the strongest interaction could be possible through the nucleophilic center on the $\mathrm{N}(\mathrm{pH})$ of $\mathrm{CV}$, forming H-bridges with the matrix components. The other modes shifts could be consequences of the binding mentioned above, like the deformation $\mathrm{Ph}-\mathrm{C}^{+}-\mathrm{Ph}$ mode.

The possible interactions between ALMP and AG in the formulation were explored by the comparison of infrared spectra of formulation and the individual gel components (ALMP and AG). To improve this analysis the resulting spectrum of the sum of ALMP and AG spectra was also considered.

The infrared spectra of ALMP, AG, the biopolymer formulation and the additive spectra of ALMP and AG are shown in Fig. 7. Table 3 is collecting the corresponding infrared bands for all the above spectra. Spectroscopic shifts, defined as: formulation wavenumber-ALMP (or -AG) wavenumbers, are also included.

As mentioned above, $\mathrm{CV}$ bands were not detected in the formulation in the infrared spectra because of its low concentration in the matrix. Then, infrared spectroscopy can be useful to detect interaction between the components of the formulation, LMPA, AG and $\mathrm{Ca}^{2+}$ ion. Raman

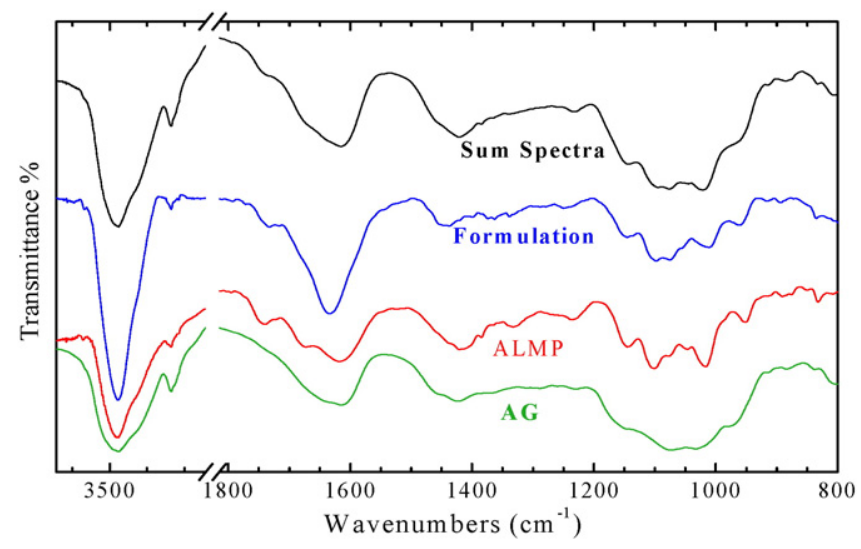

Fig. 7. FTIR spectra of ALMP, AG, formulation and sum of spectra of ALMP and AG.

spectroscopy evidenced interactions between $\mathrm{CV}$ and the remaining components.

Frequencies and relative intensities for ALMP and AG bands observed in our infrared spectra are in agreement with some of those reported before [28-33]. The bands in the $3700-3000 \mathrm{~cm}^{-1}$ region are assigned to the $v(\mathrm{OH})$ stretching vibration. Fig. 7 shows that the $v(\mathrm{OH})$ bands in the gel are thinner than in pectin and Arabic gum spectra. This is also appreciated when comparing the formulation with the sum of spectra. It could be interpreted that during the gelation process a more rigid and ordered structure is achieved with the formation of fewer hydrogen bridges, leading to a thinner vibrational band.

The band at $1741 \mathrm{~cm}^{-1}$ of ALMP was assigned to the CO stretching bands of methyl ester according to the literature sources [29]. Since the AG does not contain that functional group, the formulation band at $1734 \mathrm{~cm}^{-1}$ should come from to the contribution of the ALMP. This band shows a clear shift by $-7 \mathrm{~cm}^{-1}$ respect to the formulation suggesting a small interaction possible due to H-bridges or calcium complexes.

The bands at $1618 \mathrm{~cm}^{-1}$ and $1416 \mathrm{~cm}^{-1}$ of ALMP spectrum were assigned to asymmetric and symmetric stretching of the carboxylate

Table 3

Infrared bands of amidated low methoxylated pectin(AMLP), Arabic gum (AG) and the formulation $(\mathrm{F})$

\begin{tabular}{|c|c|c|c|c|c|}
\hline $\begin{array}{l}\text { ALMP } \\
(P)\end{array}$ & $\begin{array}{l}\text { Shift } \\
\Delta(\text { F-P })\end{array}$ & $\begin{array}{l}\text { Formulation } \\
\text { (F) }\end{array}$ & $\begin{array}{l}\text { Shift } \\
\Delta(\mathrm{F}-\mathrm{AG})\end{array}$ & $A G$ & Assignments \\
\hline $3424 v s$ & - & 3423vs & - & $3424 v s$ & $v(\mathrm{OH})_{\mathrm{CO}-\mathrm{H}}, v(\mathrm{OH})_{\mathrm{H} 2 \mathrm{O}}$ \\
\hline $3424 v s$ & - & 3423vs & - & - & $v(\mathrm{NH})$ \\
\hline 3230sh & - & 3230sh & - & 3230sh & $v(\mathrm{OH})_{-\mathrm{COO}-\mathrm{H}}$ \\
\hline $2957 \mathrm{sh}$ & - & $2956 \mathrm{sh}$ & & - & $v_{\mathrm{as}}\left(\mathrm{CH}_{3}\right)$ \\
\hline $2925 w$ & - & $2925 w$ & & $2929 w$ & $v(\mathrm{CH})_{\mathrm{OH}}$ \\
\hline & & & -4 & 2890sh & $v_{\text {as }}\left(\mathrm{CH}_{2}\right)$ \\
\hline $2855 \mathrm{vw}$ & - & $2855 \mathrm{vw}$ & & 2854 sh & $v_{\text {sim }}\left(\mathrm{CH}_{3}\right)$ \\
\hline 1741 & -7 & 1734 & - & - & $v(\mathrm{CO})_{\mathrm{COOCH} 3}$ \\
\hline 1674 & -9 & 1683(sh) & - & - & $\delta\left(\mathrm{H}_{2} \mathrm{O}\right), \delta\left(\mathrm{NH}_{2}\right)$ \\
\hline 1618 & +16 & 1634 & +21 & $\begin{array}{l}1613 \\
1460 \\
\text { sh }\end{array}$ & $v_{\mathrm{as}}\left(\mathrm{COO}^{-}\right)$ \\
\hline 1416 & +29 & 1445 & +23 & 1422 & $v_{\text {sim }}\left(\mathrm{COO}^{-}\right)$ \\
\hline 1385 & - & - & - & - & $\delta(\mathrm{CH})$ \\
\hline 1333 & - & - & - & - & $\delta(\mathrm{CH})$ \\
\hline 1234 & - & - & - & - & $\delta(\mathrm{CH})$ \\
\hline 1145 & - & 1146 & - & $\begin{array}{l}1148 \\
\text { sh }\end{array}$ & $v(\mathrm{COC})_{\text {glicos bond }}$ \\
\hline 1101 & - & 1098 & - & - & $\nu \mathrm{O}-\mathrm{CH}_{3}$ (ester), Ring \\
\hline 1077 & - & 1076 & - & 1075 & vibrations \\
\hline 1047 & - & 1055 & - & & \\
\hline 1016 & +5 & 1011 & -21 & 1032 & Gal A, $\gamma(\mathrm{COOH})_{\text {dimers }}$ \\
\hline 952 & - & 961 & - & $973 \mathrm{sh}$ & $\begin{array}{l}\text { Gal A } \delta(\mathrm{CCH}), \delta(\mathrm{COH}) \text { out of } \\
\text { plane }\end{array}$ \\
\hline 832 & - & 836 & - & 838 & $\gamma(\mathrm{COH})_{\text {ring }}$ \\
\hline
\end{tabular}


groups, respectively $[29,30]$ The corresponding bands for AG are seen at $1613 \mathrm{~cm}^{-1}$ and $1422 \mathrm{~cm}^{-1}$ [31]. Both modes in ALMP and AG are shifted to higher frequency in the gel. The shifts and the thinning observed for these bands suggest an increasing interaction, leading to a stronger bond in formulation. This behavior could be consistent with the possible complexation with calcium.

The glycosidic linkage vibrations are observed at about $1145 \mathrm{~cm}^{-1}$, the assignments of the remaining bands for the region of 1200 to $900 \mathrm{~cm}^{-1}$ are not in good agreement in the literature. Main assignments reported in the literature for this bands are: ring vibrations, $\delta(\mathrm{CCH}) ; v(\mathrm{CC}) v(\mathrm{CO}), \gamma(\mathrm{COOH})$ and $\delta(\mathrm{COH})$. The observed shifts in this region are very small except for bands at 1016 (ALMP) and $1032 \mathrm{~cm}^{-1}$ (AG).

In summary, the larger shifts observed in the matrix correspond mainly to carbonyl and carboxylate groups. We speculate possible interactions between above groups with the formation of $\mathrm{H}$ bridges because of large amount of available $\mathrm{OH}$ groups with the nucleophilic center of (C) $\mathrm{O}$ and carboxylate groups. Additionally, calcium ion could play a crucial role in above mentioned interactions by interacting with the carboxylate ions.

Aqueous solutions of $\mathrm{CV}$ in the range from $100 \mu \mathrm{M}$ to $1.0 \mathrm{mM}$ showed a characteristic peak at $592 \mathrm{~nm}\left(\bar{v}=16,900 \mathrm{~cm}^{-1}\right)$ associated with a growing shoulder alongside with the increase of dye concentration at $556 \mathrm{~nm}\left(\bar{v}=18,000 \mathrm{~cm}^{-1}\right)$ as reported earlier [17]. The shoulder was considered the $\mathrm{CV}$ aggregation, which at low dye concentration was reported as CV dimer. On the other side, the CV released from the ALMP-AG microspheres do not show the shoulder at $556 \mathrm{~nm}$, but a bathochromic shift of the dye maximum absorption from $592 \mathrm{~nm}$ to $600-602 \mathrm{~nm}$, depending of the release time, was observed. This result is suggesting that the monomer of $\mathrm{CV}$ remains associated to the polymeric chains and released from the gel to the solution. Consequently encapsulation of CV could enhance the biodisponibility of the dye by considering the mucoadhesiveness of both chosen polymers.

\subsection{CV stability studies}

CV kinetic release from ALMP-AG (2:1) gel microspheres at pH 1.2 and $37{ }^{\circ} \mathrm{C}$ could not be quantified because of the dye instability in a highly acidic medium; although the microspheres kept the morphology at least for one week. High swelling of ALMP-AG (2:1) gel microspheres crosslinked with calcium was observed at $\mathrm{pH} 3.0$, below the pectin $\mathrm{pK}_{\mathrm{a}}$ (i.e., about 3.8-4.5) [34]. The CV release from the ALMP-AG microspheres made at $\mathrm{pH} 3.0$ was $35 \%$ higher than at $\mathrm{pH} 5.0$ which could be attributed to the ionotropic effect of calcium crosslinking in the matrix strength. The comparative low stability of ALMP-AG gel matrix produced at pH 3.0 related to $\mathrm{pH} 5.0$ is indicative of the low availability of the carboxylate ions to be crosslinked with calcium ion which is reducing the matrix stability. In fact, the effect of calcium ion in the matrix crosslinking at pH 5.0 and above the pKa of ALMP (i.e., about 3.8-4.5) increases because of the carboxylic acid residues are in the anionic form. On the other hand, the polar and hydrogen bridges between the dissociated carboxylic groups and the secondary alcohols and amide groups of the ALMP and AG chains will be augmented. In addition, the polar interaction between the load and ALMP-AG should be reduced close to the minimum at $\mathrm{pH} 5.0$ considering the $\mathrm{CV} \mathrm{pK}_{\mathrm{a}}$ of 4.83 , which allows to enhance the non-ionic interactions between the dye and the matrix components, [17].

The CV kinetic release from ALMP-AG (2:1) gel microspheres made at $\mathrm{pH} 3.0$ and 5.0 were tested at $\mathrm{pH} 7.4$ and $37{ }^{\circ} \mathrm{C}$ in presence of $154 \mathrm{mM} \mathrm{NaCl}$ (e.g., physiological conditions) to analyze the influence of ionization degree of pectin and the $\mathrm{pH}$ in the coacervate gel matrix stability. The $\mathrm{CV}$ release at $\mathrm{pH} 7.4$ in presence of $154 \mathrm{mM} \mathrm{NaCl}$ at $37^{\circ} \mathrm{C}$ was similar for both type of microspheres synthesized at pH 3.0 and 5.0 and faster compared to the system without $\mathrm{NaCl}$ (Fig. 8). In both cases, the stability of the ALMP-AG matrix is not only compromised by

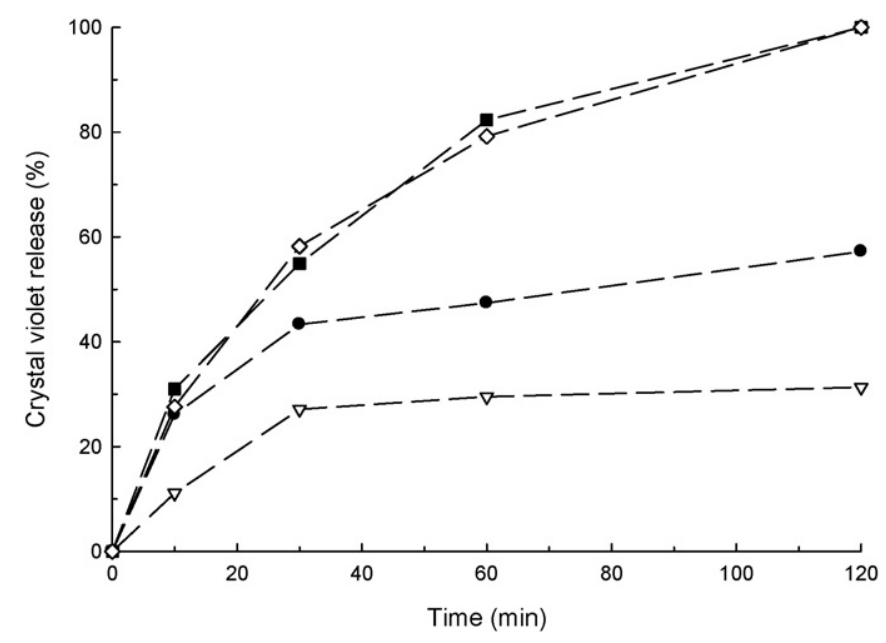

Fig. 8. Kinetic release of Crystal Violet in $100 \mathrm{mM}$ TES buffer $(\mathrm{pH}=7.4)$ form the ALMP-AG microspheres synthetized at pH $3.0(\bullet, \square)$ and $5.0(\nabla, \diamond)$ in presence $(\boldsymbol{\square}, \diamond)$ or not $(\bullet, \nabla)$ of $154 \mathrm{mM} \mathrm{NaCl}$ respectively.

the $\mathrm{Na}^{1+}$ ion diffusion gradient from the solution to the matrix but also by diffusion of calcium from the gel matrix to the solution, competing and replacing the divalent ion and consequently debilitating the gel network structure concurrently with the increase of the release rate of the $\mathrm{CV}$.

\section{Conclusions}

The ionotropic gelation of ALMP-AG coacervate allowed to encapsulate $\mathrm{CV}$, making an stable gel matrix with reproducible morphology and high mechanical resistance. The rheological behavior of the matrix components (i.e., ALMP and AG) and the microspheres texture not only allow to select the optimal gel conditions but also to produce macroscopic information of the ALMP-AG matrix with and without CV. The FTIR and Raman spectroscopies are both bringing complementary information about the matrix components and the cargo playing a crucial role in determining the main interactions between ALMP, AG and the CV.

The main advantage of the ALMP-AG biopolymeric matrix is the possibility of making a tailored modular gel system for encapsulation of molecules with different properties. The ALMP-AG gel matrix is not only acting as physical support of the load, but also the chemical interactions between the matrix components and the CV are able to modulate the molecular release and consequently the biodisponibility of the load under different environmental conditions. Characterization studies of the individual matrix components-load by biophysical methods allow to establish the type of molecular interactions and controlled it by changing the biomatrix composition, and/or chemical structure by biocatalysis and/or chemical methods.

\section{Acknowledgments}

Support from CONICET, Universidad Nacional de La Plata and ANPCyT (PIP-0214, X/545, and PICT2011-2116 respectively) from Argentina to GRC; and from CONICET (PIP-0327), Universidad Nacional de Luján and Universidad Nacional de La Plata to JAG is gratefully acknowledged. Dr. MEChV is Professional Staff of Comisión de Investigaciones Científicas de la Provincia de Buenos Aires (CICPBA), Argentina. 


\section{Appendix A. Supplementary data}

Supplementary data to this article can be found online at http://dx. doi.org/10.1016/j.reactfunctpolym.2016.07.002.

\section{References}

[1] F. Nazzaro, P. Orlando, F. Fratianni, R. Coppola, Curr. Opin. Biotechnol. 23 (2012) $182-186$.

[2] D.J. McClements, Nanoparticle- and Microparticle-based Delivery Systems, CRC Press, Boca Raton, Fl, 2015.

[3] P. Mura, N. Mennini, I. Kosalec, S. Furlanetto, S. Orlandini, M. Jug, Carbohydr. Polym. 121 (2015) 231-240.

[4] E. da S. Gulão, C.J.F. de Souza, C.T. Andrade, E.E. García-Rojas, Food Chem. 194 (2016) 680-686.

[5] G. Phillips, P. Williams, Food Hydrocoll. 19 (2005) 661-667.

[6] A. Madene, M. Jacquot, J. Scher, S. Desobry, Int. J. Food Sci. Technol. 41 (2006) 1-21.

[7] G.A. Islan, A. Mukherjee, G.R. Castro, Int. J. Biol. Macromol. 72 (2015) 740-750.

[8] R. Docampo, S.N.J. Moreno, Drug Metab. Rev. 22 (1990) 161-178.

[9] Z. Chen, L. Hu, M.J. Serpe, J. Mater. Chem. 22 (2012) 20998-21002.

[10] G. Jones II, C. Oh, K. Goswami, J. Photochem. Photobiol. A Chem. 57 (1991) 65-80.

[11] O.S. Amodu, T.V. Ojumu, S.K. Ntwampe, O.S. Ayanda, J. Encap. Adsorpt. Sci. 5 (2015) 191-203.

[12] M. Perullini, N. Levinson, M. Jobbágy, S.A. Bilmes, J. Sol-Gel Sci. Technol. 77 (2016) 437-445.

[13] S. Kipke, G. Schmid, 14 (2004) 1184-1188.

[14] S. Naeem, L.V. Kiew, L.Y. Chung, M.A. Ashraf, M. Bin Misran, Sains Malays 45 (2016) 71-77.

[15] Z. Liu, Y. Jiao, Y. Wang, C. Zhou, Z. Zhang, Adv. Drug Deliv. Rev. 60 (2008) 1650-1662.
[16] L.G. Santiago, G.R. Castro, Curr. Op. Food Sci. 7 (2016) 78-85.

[17] W.H.J. Stork, G.J.M. Lippits, M. Mandel, J. Phys. Chem. 76 (1972) 1772-1775.

[18] R.W. Scott, Anal. Chem. 51 (1979) 936-941.

[19] J. Steffe, Rheological Methods in Food Process Engineering, second ed. Freeman Press, East Lansing, MI, EE.UU, 1996.

[20] V. Evageliou, G. Tseliou, L. Mandala, M. Komaitis, J. Food Eng. 101 (2010) 381-385.

[21] Z. Wakerly, J. Fell, D. Attwood, D. Parkins, J. Pharm. Pharmacol. 49 (1997) 622-625.

[22] C. Sanchez, D. Renard, P. Robert, C. Schmitt, J. Lefebvre, Food Hydrocoll. 16 (2002) 257-267.

[23] S. Yoo, M. Fishman, A. Hotchkiss, H. Gyu Lee, Food Hydrocoll. 20 (2006) 62-67.

[24] Y. Dror, Y. Cohen, R. Yerushalmi-Rozen, J. Polym. Sci. B Polym. Phys. 44 (2006) 3265-3271.

[25] E.J. Liang, X.L. Ye, W. Kiefer, J. Phys. Chem. A 101 (1997) 7330-7335.

[26] V.E. Bosio, B. Sreyasree, A. Fraqooue, M.E. Chacon Villalba, J.A. Güida, A. Mukherjee, G.R. Castro, React. Funct. Polym. 82 (2014) 103-110.

[27] S. Lovell, B.J. Marquardt, B. Kahr, J. Am. Chem. Soc. 2 (1999) 2241-2247.

[28] D. Lin-Vien, N.B. Colthup, W.G. Fateley, J.G. Grasselli, The Handbook of Infrared and Raman Characteristic Frequencies of Organic Molecules, Academic Press, New York, 1991.

[29] A. Synytsya, J. Copikova, P. Matejka, V. Machovic, Carbohydr. Polym. 54 (2003) 97-106.

[30] M.H. Ropers, A. Meister, A. Blume, M.C. Ralet, Biomacromolecules 9 (2008) 1306-1312.

[31] N.A. Almuslet, E.A. Hassan, A.S. Abd-El-Magied, A. Sherbini, M.G. Alla Muhgoub, J. Phys. Sci. 23 (2012) 43-53.

[32] N. Wellner, M. Kacurakova, A. Malovikova, R.H. Wilson, P.S. Belton, Carbohydr. Res. 308 (1998) 123-131.

[33] B. Smith, Infrared Spectral Interpretation, CRC Press, Boca Raton, 1999

[34] C. Löfgren, S. Guillotin, A.M. Hermansson, Biomacromolecules 7 (2006) 114-121. 